\section{Prevalência de insegurança alimentar em Pelotas, Rio Grande do Sul, Brasil, e estado nutricional de indivíduos que vivem nessa condição}

\author{
Prevalence of food insecurity in Pelotas, \\ Rio Grande do Sul State, Brazil, and \\ associated nutritional status
}

1 Centro de Pesquisas Epidemiológicas, Universidade Federal de Pelotas, Pelotas, Brasil.

2 Faculdade de Nutrição, Universidade Federal de Pelotas, Pelotas, Brasil.

Correspondência J. V. Santos

Centro de Pesquisas Epidemiológicas,

Universidade Federal de Pelotas.

Rua Marechal Deodoro 1160, 3 o andar, Pelotas, $R S$ 96020-220, Brasil. janavieira@terra.com.br

\begin{abstract}
A population-based cross-sectional study was conducted in Pelotas, Southern Brazil, to assess the prevalence of food insecurity and describe the nutritional status of family members living in this condition. The sample included 1,450 households from the urban area of Pelotas. The six questions of the Food Insecurity Scale (United States Department of Agriculture) were administered to the person in charge of preparing meals in the house. A 12-month recall period was used. If the household was considered insecure, all members were weighed and measured. Prevalence of food insecurity for all families was 11\% (95\% CI: 9\%-13\%). In families living in food insecurity, overweight and obesity were frequent; meanwhile, $21 \%$ of the children showed height deficit. The authors conclude that food insecurity results not only from quantitative limitations but also from deficient food quality.
\end{abstract}

Food Habits; Nutritional Status; Food Security
Janaína Vieira dos Santos 1 Denise Petrucci Gigante 2 Marlos Rodrigues Domingues 1

\section{Introdução}

Grande parte dos problemas pelos quais passam parcelas significativas da população mundial está relacionada com questões alimentares e nutricionais, seja pela ausência do alimento, pela má qualidade da alimentação ou por condições de vida e saúde que impedem o aproveitamento adequado do alimento disponível 1 .

Segurança alimentar e nutricional é a realização do direito de todos ao acesso regular e permanente a alimentos de qualidade, em quantidade suficiente, sem comprometer o acesso a outras necessidades essenciais, tendo como base práticas alimentares promotoras de saúde, que respeitem a diversidade cultural e que sejam social, econômica e ambientalmente sustentáveis 2,3,4.

Para promover a segurança alimentar a medida de insegurança tem sido proposta no monitoramento de iniqüidades, podendo ser complementar ao conjunto de indicadores sociais ou utilizada de forma isolada para identificar grupos vulneráveis em que a preocupação e a angústia da incerteza de dispor regularmente de comida até a falta de alimento podem estar presentes 5 .

Em 1989, nos Estados Unidos, foi desenvolvido um instrumento capaz de dar uma dimensão à magnitude da insegurança alimentar. Essa escala já sofreu várias modificações e hoje existem versões curtas e outras mais completas 2,6. Vários países no mundo validaram algum instrumento baseado nessas escalas 7,8,9,10,11. Esses instru- 
mentos avaliam insegurança alimentar através da percepção das famílias, não contemplando o conceito de segurança alimentar como um todo, apenas o acesso aos alimentos.

No Brasil o instrumento baseado na escala americana, na versão longa, é a Escala Brasileira de Insegurança Alimentar (EBIA) 12 e foi utilizada pela Pesquisa Nacional por Amostra de Domicílios (PNAD) 13, realizada em 2004, estimando que cerca de $30 \%$ dos domicílios particulares da zona urbana do Brasil viviam em situação de insegurança alimentar. Para o Estado do Rio Grande do Sul, resultados desse mesmo estudo mostraram prevalência em torno de 25\%. Em 2006, a Pesquisa Nacional de Demografia e Saúde da Criança $e$ da Mulher (PNDS) 14 confirmou esses dados, mostrando que $32 \%$ das famílias residentes na zona urbana do país estavam em insegurança alimentar e, para a Região Sul, a prevalência foi semelhante àquela encontrada dois anos antes para o Rio Grande do Sul.

No Brasil, assim como em vários outros países menos desenvolvidos, a coexistência de déficit nutricional com excesso de peso, assim como da carência de micronutrientes com doenças crônicas não transmissíveis nas mesmas regiões - e por vezes dentro do mesmo domicílio - caracteriza a chamada transição nutricional 15 .

Na Pesquisa de Orçamentos Familiares (POF), realizada em 2002, o estado nutricional da população brasileira foi avaliado. Para os adultos da zona urbana da Região Sul o déficit de peso foi $2 \%$ para os homens e $4 \%$ para as mulheres. As prevalências de déficit nutricional em adultos foram semelhantes para o país como um todo 16 . Para crianças e adolescentes o déficit em estatura foi o mais prevalente, enquanto o déficit de peso nas crianças não atinge $5 \%$. Por outro lado, as prevalências de excesso de peso e obesidade, embora mais elevadas na população adulta, atingem parcela importante de crianças e adolescentes. A comparação dos resultados da POF com outros estudos de abrangência nacional realizados anteriormente mostram que enquanto o déficit nutricional vem diminuindo, as prevalências de excesso de peso e obesidade aumentaram em todos os grupos etários 16,17.

O presente estudo tem como objetivo apresentar a prevalência de insegurança alimentar em famílias da cidade de Pelotas, Rio Grande do Sul, bem como descrever o estado nutricional dos membros dessas famílias em insegurança alimentar.

\section{Metodologia}

Estudo transversal de base populacional realizado em Pelotas, município com população estimada de 340 mil habitantes, sendo 93\% moradores na zona urbana.

Para o cálculo do tamanho da amostra utilizou-se a prevalência esperada de insegurança alimentar nas famílias de Pelotas de $24 \%$, nível de $95 \%$ de confiança e erro aceitável de quatro pontos percentuais. A este número foram acrescidos $10 \%$ para perdas e recusas e multiplicou-se por 1,5 para compensar possível efeito de delineamento amostral, visto que a amostragem foi por conglomerados. Assim, 1.275 famílias seria o número necessário para determinação da prevalência de insegurança alimentar em Pelotas.

A seleção da amostra foi em múltiplos estágios, baseada nos dados do censo populacional brasileiro do ano 2000, realizado pelo Instituto Brasileiro de Geografia e Estatística (IBGE; http://www.ibge.gov.br). Cada setor censitário da cidade, do total de 404, foi listado em ordem crescente de renda média dos chefes de família. A seguir, selecionaram-se 126 setores por sorteio sistemático, com probabilidade proporcional ao tamanho. Na etapa seguinte visitaram-se todos os domicílios de cada setor selecionado, identificando-se aqueles habitados ou de comércio. A partir da lista de domicílios habitados, fez-se um sorteio sistemático proporcional ao tamanho, selecionando-se, em média, dez casas em cada setor ( $\mathrm{n}=1.512$ domicílios). Essas foram visitadas pelo pesquisador para entrega de carta de apresentação e obtenção da informação sobre o número, idade e sexo dos moradores no domicílio. Nesse momento, foram fornecidos esclarecimentos sobre o estudo, solicitando que recebessem a entrevistadora e participassem da pesquisa. Esses domicílios foram então visitados e o questionário de insegurança alimentar aplicado ao responsável pela alimentação no domicilio por entrevistadoras treinadas. Tais questionários haviam sido testados em estudo-piloto conduzido em setor censitário não sorteado para participar da amostra. Essa fase do trabalho de campo ocorreu no período de outubro de 2007 a janeiro de 2008.

Os domicílios em situação de insegurança alimentar identificados na primeira fase do estudo receberam uma segunda visita, quando todos os membros da família tiveram o peso e a estatura aferidos por estudantes universitários de cursos da área da saúde, treinados de acordo com técnicas padronizadas 18 . Essa segunda fase ocorreu entre fevereiro e junho de 2008, quando 110 domicílios foram visitados e todos os seus integrantes medidos. Para a obtenção dessas me- 
didas utilizaram-se balanças portáteis da marca SECA (UNICEF, Copenhagen, Dinamarca) com capacidade de $150 \mathrm{~kg}$ e precisão de $100 \mathrm{~g}$ e estadiômetro Alturexata (Alturexata, Belo Horizonte, Brasil), com escala de 35 a $213 \mathrm{~cm}$ e precisão de $0,1 \mathrm{~cm}$.

A variável "insegurança alimentar" foi obtida através de versão curta da Escala de Segurança Alimentar, do United States Department of Agriculture (USDA) 2,19 - que inclui seis questões aplicadas ao responsável pela alimentação no domicílio referente aos 12 meses anteriores à entrevista (Tabela 1). Essa escala gera um escore que varia de 0 a 6 . Em quatro questões cada resposta positiva corresponde a um ponto, enquanto em uma das questões o ponto foi dado na resposta negativa e em outra, a pontuação corresponde ao tempo de exposição em que houve diminuição na quantidade de comida por falta de dinheiro. O escore é calculado pela soma desses pontos. Considera-se como segurança para aquelas famílias que atingem um ponto; insegurança sem fome para aquelas que somam entre dois e quatro pontos e com fome quando o escore atinge cinco ou seis pontos.

As variáveis independentes incluíram sexo do chefe da família (homem, mulher ou ambos); escolaridade da pessoa de maior renda $(<4,4-7$, 8-10, 11-14, $\geq 15$ anos completos de estudo); número de moradores no domicílio (ordinal em 5 categorias); classe econômica de acordo com critério da Associação Brasileira de Estudos e Pesquisas - ABEP (ordinal em 5 categorias) e renda familiar em salários mínimo. Para a descrição do estado nutricional dos indivíduos que vivem nos domicílios em situação de insegurança alimentar utilizou-se a classificação da Organização Mundial da Saúde (OMS) 20,21,22 com base no índice de massa corporal (IMC) obtido pela divisão do peso (em quilogramas) pela altura (em metros) elevada ao quadrado. Foram classificados com déficit nutricional crianças e adolescentes com IMC inferior a - 2 escores $\mathrm{z}$ da curva de referência da OMS e adultos com IMC inferior a $18,5 \mathrm{~kg} / \mathrm{m}^{2}$. Excesso de peso foi definido para crianças e adolescentes que apresentavam IMC superior a 1 escore $\mathrm{z}$ da curva de referência ou para adultos quando era igual ou superior a $25 \mathrm{~kg} / \mathrm{m}^{2}$. IMC superior a 2 escores $\mathrm{z}$ da curva de referência definiu obesidade para crianças e adolescentes, enquanto IMC igual ou superior a $30 \mathrm{~kg} / \mathrm{m}^{2}$ foi utilizado para adultos.

Além da classificação através do IMC, os indivíduos menores de 19 anos foram também avaliados segundo o índice antropométrico estatura-para-idade (E/I) de acordo com o sexo e faixa etária. Foram classificados como déficit aqueles que apresentaram valores iguais ou inferiores a
Tabela 1

Questões utilizadas na versão curta da Escala de Segurança Alimentar.

\section{Questões}

Alguma vez terminou a comida da casa e o(a) Sr.(a) não tinha dinheiro para comprar mais? O(A) Sr.(a) pode oferecer uma alimentação variada, com feijão, arroz, carne, saladas e frutas para sua família?

$\mathrm{O}(\mathrm{A}) \mathrm{Sr}$.(a) ou alguma outra pessoa na sua casa tiveram que diminuir a quantidade de comida ou não fazer alguma refeição por falta de dinheiro para

comprar mais? Em quantos meses isso aconteceu?

$\mathrm{O}(\mathrm{A}) \mathrm{Sr}$.(a) comeu menos do que gostaria porque não tinha dinheiro para comprar mais?

$\mathrm{O}(\mathrm{A}) \mathrm{Sr}$.(a) sentiu fome, mas não comeu porque não tinha dinheiro para comprar mais comida?

Fonte: Bickel et al. 2 .

-2 escores $\mathrm{z}$ de referência para o índice E/I 22. A entrada de dados foi feita com digitação dupla, com checagem de inconsistências e amplitudes, sendo utilizado o programa Epi Info 6.04 (Centers for Disease Control and Prevention, Atlanta, Estados Unidos). A análise dos dados foi feita no programa Stata, versão 9.0 (Stata Corp., College Station, Estados Unidos), sendo que proporções e seus respectivos intervalos de confiança foram utilizados na análise descritiva. As prevalências de insegurança alimentar de acordo com as categorias das variáveis independentes foram avaliadas através do teste do qui-quadrado (heterogeneidade ou tendência linear) e o nível de significância utilizado foi de $5 \%$ para todos os testes bi-caudais.

A pesquisa foi aprovada pelo Comitê de Ética em Pesquisa da Faculdade de Medicina da Universidade Federal de Pelotas e todos os participantes assinaram um termo de consentimento informado.

\section{Resultados}

Dos 1.512 domicílios identificados, 1.450 foram estudados, representando 95,3\% do total. As perdas e recusas distribuíram-se igualmente entre os setores censitários.

Na Tabela 2 observa-se a distribuição das famílias em relação às variáveis sócio-demográficas. Há um predomínio das famílias em que o chefe era do sexo masculino, a pessoa de maior renda apresentava entre 4 e 7 anos de escolaridade, pertencentes à classe social C de acordo com os critérios da ABEP, possuíam três membros em seu domicílio e recebiam entre 2 e 4 salários mí- 
Tabela 2

Distribuição de uma amostra de famílias de acordo com características sócio-econômicas e demográficas. Pelotas, Rio Grande do Sul, Brasil, 2007.

\begin{tabular}{|c|c|c|}
\hline Variáveis & $\mathrm{n}$ & $\%$ \\
\hline \multicolumn{3}{|l|}{ Chefe da família * } \\
\hline Homem & 538 & 39,4 \\
\hline Mulher & 480 & 35,1 \\
\hline Ambos & 348 & 25,5 \\
\hline \multicolumn{3}{|c|}{ Escolaridade da pessoa de maior renda (anos) ${ }^{* \star}$} \\
\hline$>4$ & 217 & 15,0 \\
\hline $4-7$ & 408 & 28,3 \\
\hline $8-10$ & 240 & 16,6 \\
\hline $11-14$ & 352 & 24,3 \\
\hline 15 ou mais & 229 & 15,8 \\
\hline \multicolumn{3}{|c|}{ Classificação econômica (ABEP) ** } \\
\hline A & 82 & 5,7 \\
\hline B & 438 & 30,4 \\
\hline $\mathrm{C}$ & 611 & 42,3 \\
\hline $\mathrm{D}$ & 278 & 19,3 \\
\hline$E$ & 33 & 2,3 \\
\hline \multicolumn{3}{|l|}{ Número de moradores } \\
\hline 1 & 234 & 16,1 \\
\hline 2 & 350 & 24,1 \\
\hline 3 & 377 & 26,0 \\
\hline 4 & 272 & 18,8 \\
\hline 5 & 127 & 8,8 \\
\hline 6 ou mais & 90 & 6,2 \\
\hline \multicolumn{3}{|c|}{ Renda familiar (salários mínimos) * } \\
\hline$>2$ & 387 & 28,6 \\
\hline $2-3,9$ & 499 & 36,8 \\
\hline 4 ou mais & 469 & 34,6 \\
\hline \multicolumn{3}{|c|}{ Nível de insegurança alimentar } \\
\hline Segurança & 1.288 & 89,0 \\
\hline Insegurança sem fome & 117 & 8,1 \\
\hline Insegurança com fome & 42 & 2,9 \\
\hline Total & 1.450 & 100,0 \\
\hline
\end{tabular}

ABEP: Associação Brasileira de Estudos e Pesquisas.

* Nessas variáveis houve até $7 \%$ de perdas de informação;

** Nessas variáveis houve até $1 \%$ de perdas de informação.

nimos. A média de moradores nos domicílios foi três (DP 1,6), com amplitude variando entre $1 \mathrm{e}$ 11 pessoas por domicílio.

A prevalência de insegurança alimentar familiar foi de $11 \%$ (IC95\%: 9,3-12,6), sendo que 3\% foram classificados em insegurança alimentar com fome. O efeito do delineamento amostral encontrado neste estudo para a prevalência de insegurança alimentar de 1,55 foi considerado no cálculo dos intervalos de confiança e análises subseqüentes.
As prevalências de insegurança alimentar agrupando a classificação com e sem fome, foram diferentes de acordo com as características sócio-demográficas (Tabela 3). Enquanto essas prevalências foram em torno de $9 \%$ nas famílias em que havia um homem como chefe da família, insegurança alimentar ocorreu em 16\% nas famílias cujo chefe era uma mulher $(p=0,4)$. Ao analisar a variável de forma dicotômica encontrou-se diferença significativa, sendo a prevalência de insegurança alimentar de $16 \%$ quando apenas uma 
Prevalência de insegurança alimentar, em uma amostra de famílias da população de Pelotas, Rio Grande do Sul, Brasil, de acordo com características sócio-econômicas e demográficas, 2007.

\begin{tabular}{|c|c|c|}
\hline Variáveis & Prevalência (\%) & IC95\% \\
\hline Chefe da família & & $p=0,4$ * \\
\hline Masculino & 8,7 & $6,4-11,8$ \\
\hline Feminino & 15,7 & $12,4-19,7$ \\
\hline Ambos & 9,3 & $6,6-12,9$ \\
\hline Escolaridade da pessoa de maior renda (anos) & & $p<0,001 * *$ \\
\hline$>4$ & 19,8 & $14,9-25,8$ \\
\hline $4-7$ & 16,3 & $12,8-20,5$ \\
\hline $8-10$ & 10,0 & $6,6-14,9$ \\
\hline $11-14$ & 6,5 & $4,3-9,7$ \\
\hline 15 ou mais & 1,3 & $0,4-4,1$ \\
\hline Classificação econômica (ABEP) & & $p<0,001 * \star$ \\
\hline A & - & - \\
\hline$B$ & 1,6 & $0,8-3,2$ \\
\hline C & 8,7 & $6,6-11,3$ \\
\hline$D$ & 27,9 & $22,9-33,6$ \\
\hline$E$ & 63,6 & $42,5-80,6$ \\
\hline Número de moradores & & $p=0,03 * \star$ \\
\hline 1 & 9,4 & $6,2-14,0$ \\
\hline 2 & 10,0 & $7,0-14,2$ \\
\hline 3 & 9,8 & $7,3-13,2$ \\
\hline 4 & 10,3 & $7,3-14,4$ \\
\hline 5 & 17,1 & $11,6-24,5$ \\
\hline 6 ou mais & 21,4 & $13,4-32,2$ \\
\hline Renda familiar (salários mínimos) & & $p<0,001 * *$ \\
\hline$>2$ & 27.7 & $23,3-32,6$ \\
\hline $2-3,9$ & 6.8 & $4,8-9,6$ \\
\hline 4 ou mais & 2.8 & $1,6-4,7$ \\
\hline
\end{tabular}

ABEP: Associação Brasileira de Estudos e Pesquisas.

* Teste de heterogeneidade de Wald;

** Teste de tendência linear.

mulher era chefe da família e, essa proporção foi de $9 \%$ quando havia pelo menos um homem $(\mathrm{p}<0,001)$.

Em relação à escolaridade da pessoa de maior renda, a insegurança alimentar foi mais prevalente nas famílias em que essa pessoa apresentou escolaridade inferior a quatro anos, sendo que nesse grupo a prevalência foi quase 20 vezes maior do que naquele cuja pessoa de maior renda tinha 11 ou mais anos de escolaridade $(\mathrm{p}<0,001)$. A maior prevalência de insegurança alimentar foi observada nas famílias com classificação econômica $\mathrm{E}$, sendo que nenhuma família com classificação econômica A apresentou insegurança alimentar, e nos domicílios com seis ou mais moradores a prevalência foi em torno de $22 \%$ ( $p<0,001)$. A prevalência de insegurança alimentar foi 10 vezes maior em famílias que receberam menos de dois salários mínimo em relação àquelas que receberam 4 ou mais salários.

Na segunda fase do trabalho 132 famílias em insegurança alimentar - 90 crianças, 78 adolescentes e 244 adultos - tiveram o peso e a altura aferidos, correspondendo a $83 \%$ do total de pessoas das famílias em insegurança alimentar. De acordo com o índice de massa corporal (Figura 1), a prevalência de obesidade foi mais elevada nos adultos do que em adolescentes e crianças. Cabe destacar que mesmo entre as crianças a prevalência de obesidade foi também elevada (15\%; IC95\%: 7,7-23,9). Enquanto 31\% (IC95\%: 18,6$47,6)$ das mulheres adultas eram obesas, essa prevalência foi de 12\% (IC95\%: 0,2-38,4) para homens adultos. $\mathrm{O}$ excesso de peso também foi 
Figura 1

Estado nutricional de acordo com índice de massa corporal em crianças, adolescentes e adultos que vivem em situação de insegurança alimentar em uma amostra de famílias da cidade de Pelotas, Rio Grande do Sul, Brasil, 2007.

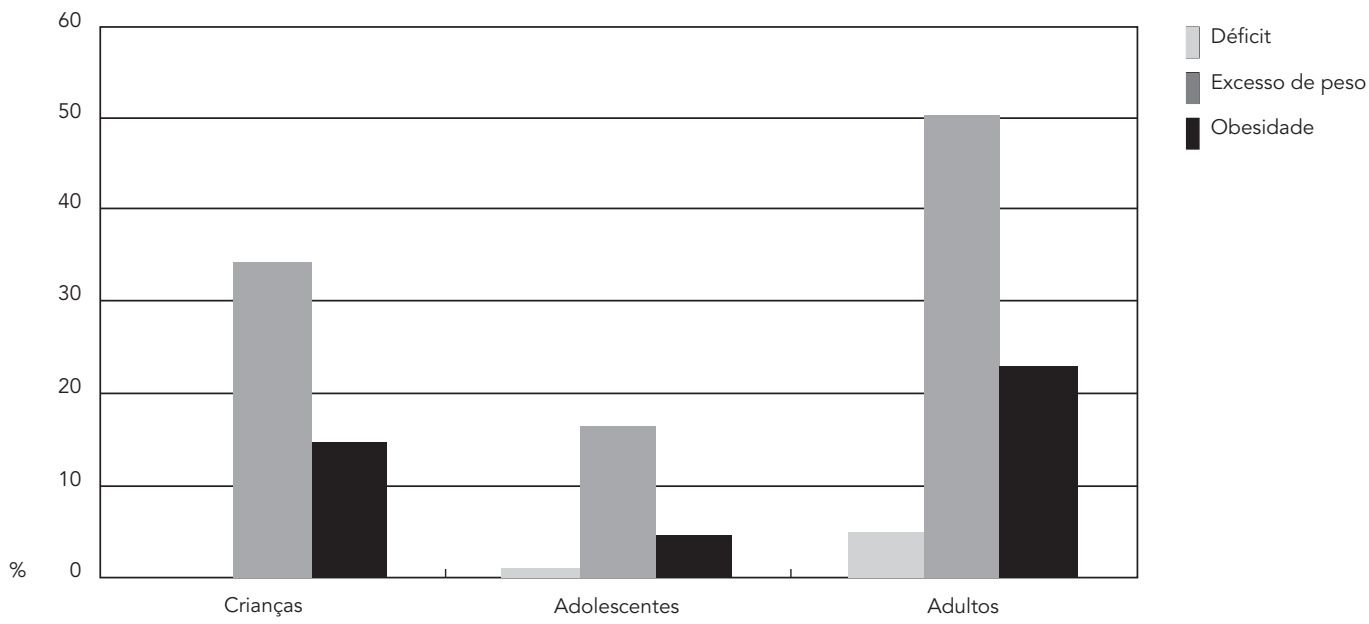

mais prevalente nos adultos, sendo maior nas mulheres (59\%; IC95\%: 50,6-67,3) do que nos homens (38\%; IC95\%: 28,8-47,5).

Das 90 crianças, 39 eram menores de cinco anos e tiveram o seu estado nutricional também avaliado através de escore $\mathrm{z}$ do índice estatura/ idade. Na Figura 2, nota-se que a prevalência de déficit em estatura foi $21 \%$ (IC95\%: 9,3-36,5) nas crianças menores de cinco anos, e de $8 \%$ para crianças maiores e adolescentes.

\section{Discussão}

O presente estudo investigou a ocorrência de insegurança alimentar em famílias residentes na zona urbana do Município de Pelotas, através de um inquérito de base populacional em que distintas variáveis sócio-demográficas foram avaliadas. O baixo percentual de perdas e sua distribuição de forma homogênea entre os diferentes setores censitários selecionados na zona urbana do município também deve ser considerado como uma vantagem para o estudo.

$\mathrm{O}$ instrumento utilizado, incluindo seis questões, é proposto pelo USDA como uma ferramenta de rastreamento para avaliar insegurança alimentar. Nos Estados Unidos foi comparado com outra escala contendo 18 questões e sua utilização recomendada, sem que se possa avaliar os níveis mais severos de insegurança alimentar. A utilização desse instrumento torna impossível as comparações dos níveis de insegurança alimentar entre as duas escalas, a versão curta utilizada no trabalho e a EBIA, mas a comparação da presença ou não de insegurança alimentar pode ser feita ${ }^{2}$.

Outra limitação do presente estudo referese à impossibilidade de comparar o estado nutricional das famílias com ou sem insegurança alimentar, uma vez que esse foi medido apenas naquelas famílias que foram classificadas em insegurança alimentar de acordo com o instrumento de rastreamento com seis questões. No entanto, a impossibilidade de comparação interna não inviabiliza que os resultados da avaliação nutricional possam ser descritos para o grupo em insegurança alimentar.

Conforme comentado anteriormente, o Brasil é carente de estudos que descrevam a situação nutricional das famílias em insegurança alimentar. Em outros países, como Estados Unidos e Canadá, vários estudos têm comparado a situação nutricional de famílias com ou sem insegurança alimentar 23,24,25,26,27.

O resultado do presente estudo está de acordo com o que seria esperado: famílias em condições sócio-econômicas menos favorecidas apresentaram maior prevalência de insegurança alimentar. A prevalência de $11 \%$ é menos da metade daquela encontrada em 2004 pela PNAD para os domicílios urbanos do Rio Grande do Sul (24,8\%) 13 
Estado nutricional de acordo com déficit de estatura/idade em crianças e adolescentes que vivem em situação de insegurança alimentar em uma amostra de famílias da cidade de Pelotas, Rio Grande do Sul, Brasil, 2007.

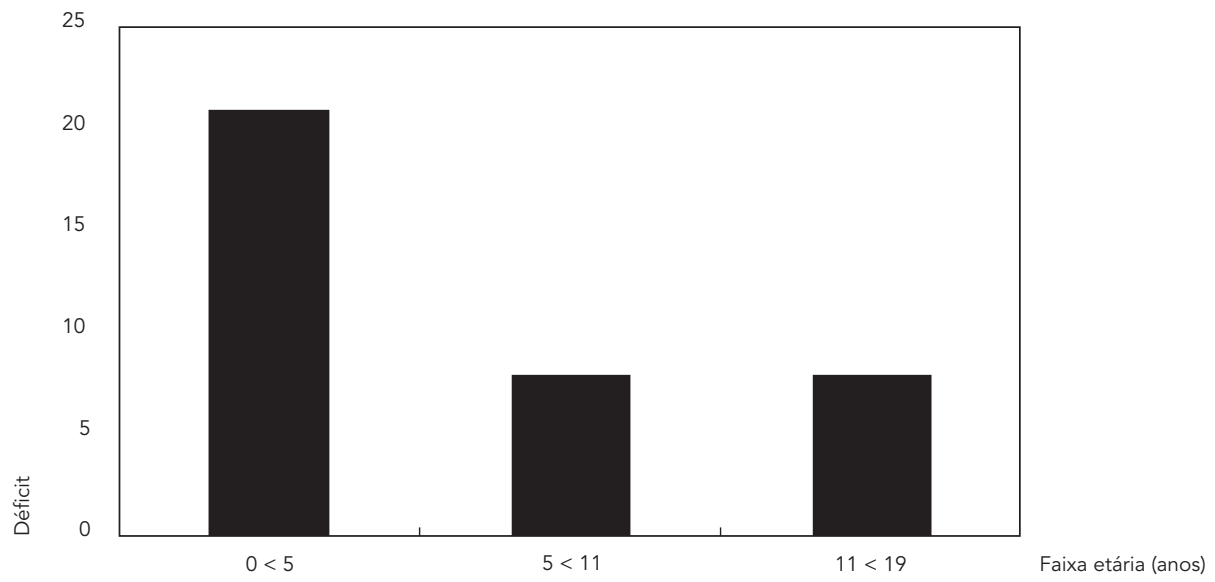

ou pela PNDS, em 2006, para a Região Sul do país $(25,7 \%)^{14}$. Cabe destacar que enquanto a PNAD e o presente estudo utilizaram processos amostrais em que a unidade de análise foi todas as famílias sorteadas nos conglomerados selecionados para os estudos, o processo da PNDS foi semelhante, porém incluiu somente famílias com mulheres de 15 a 49 anos.

Para comparar as condições sócio-econômicas das famílias do presente estudo com aquelas dos inquéritos de abrangência nacional, a distribuição de renda familiar mostra que, enquanto $6 \%$ da população de Pelotas receberam menos de um salário mínimo no mês anterior à entrevista, a proporção de famílias brasileiras que recebem menos de um salário é de $12 \%$, embora as categorias superiores tenham mostrado distribuição semelhante 13. Cabe destacar que é no grupo de menor renda que são encontradas as maiores prevalências de insegurança alimentar, o que poderia estar parcialmente ou totalmente explicando as diferentes prevalências encontradas no presente estudo e na PNAD. Em relação ao número de moradores, enquanto $40 \%$ das famílias pelotenses são constituídas por até dois membros, essa proporção é de $31 \%$ nas famílias incluídas na PNDS, e por outro lado, famílias mais numerosas foram mais freqüentes na PNDS do que em Pelotas $(21 \%$ e $15 \%$, respectivamente, com cinco ou mais membros). Considerando que as prevalências de insegurança alimentar são consideravelmente mais elevadas entre as famílias com cinco ou mais membros, a aglomeração no domicílio também poderia parcialmente explicar as diferenças encontradas.

Além da clara associação inversa entre as prevalências de insegurança alimentar e as variáveis sócio-econômicas encontradas neste estudo, outro resultado que chama atenção é a elevada prevalência de insegurança alimentar entre as famílias cujo chefe é uma mulher. Esse achado já foi apresentado em outros trabalhos 13,14. Entretanto, no presente estudo, a força dessa associação foi quase duas vezes maior para as famílias cuja mulher era chefe do que naquelas em que ambos ou sexos ou somente homens se consideravam como chefes da família.

A aglomeração no domicílio é outra variável que tem sido relacionada à insegurança alimentar. Entretanto, enquanto os resultados dos estudos de abrangência nacional mostram que a prevalência de insegurança alimentar é quase duas vezes mais elevada naquelas famílias com sete ou mais membros, quando comparadas àquelas com até três ou até quatro membros, no presente estudo a prevalência de insegurança alimentar nas famílias com sete integrantes é cerca de três vezes maior do que naquelas famílias com até três 13 ou quatro membros ${ }^{14}$, sugerindo que em Pelotas a insegurança alimentar nos domicílios estaria mais relacionada à aglomeração familiar.

Ao avaliar o estado nutricional dos adultos e adolescentes das famílias em insegurança ali- 
mentar, os resultados do presente estudo estão de acordo com o que tem sido apresentado nos Estados Unidos 23,25,26,27. Enquanto o déficit nutricional é pouco prevalente, as prevalências de excesso de peso e obesidade são bastante elevadas, especialmente na população adulta.

Nos resultados do presente estudo chama atenção que embora o déficit de peso em relação à altura tenha sido pouco freqüente nas crianças de famílias em insegurança alimentar (5\%), o déficit em estatura ocorreu em uma de cada cinco crianças de 0 a 5 anos dessas famílias 28 . Cabe destacar que a prevalência de déficit em estatura encontrada no presente estudo é mais elevada do que prevalência nacional encontrada pela PNDS (7\%), sendo superior até mesmo a prevalência encontrada na região $(14,8 \%)$, onde este déficit é mais prevalente 14 . E ainda a prevalência de déficit em estatura na Região Sul para as crianças menores de cinco anos foi semelhante àquela encontrada para crianças maiores e ado- lescentes no presente estudo. Outro resultado que merece destaque é o fato de que um entre cada dois adultos dos domicílios em insegurança alimentar tinha excesso de peso. Essa proporção é semelhante ao que foi observado na população adulta de Pelotas (53,1\%; IC95\%: 50,9-55,3) 29.

Concluindo, os resultados do presente estudo mostram que uma entre cada dez famílias residentes na zona urbana de Pelotas vive em estado de insegurança alimentar e, como seria esperado, que as características sócio-demográficas foram bastante relacionadas a esse estado. Entretanto, deve ser comentado que, entre essas famílias, a insegurança alimentar poderia estar relacionada não somente à diminuição da quantidade de alimentos como à perda da qualidade nutritiva, uma vez que o excesso de peso e a obesidade foram mais freqüentes principalmente entre os adultos, e que o déficit em estatura ainda é bastante prevalente nas crianças menores de cinco anos de idade dessas famílias.

\section{Resumo}

Um estudo transversal de base populacional foi realizado em Pelotas, Sul do Brasil, para determinar a prevalência de insegurança alimentar, além de descrever o estado nutricional dos membros das famílias que vivem nessa condição. A amostra incluiu 1.450 domicílios da área urbana de Pelotas. Nessas famílias, foi aplicada uma versão curta da Escala de Segurança Alimentar (United States Department of Agriculture) - seis questões aplicadas ao responsável pela alimentação no domicílio referente aos 12 meses anteriores à entrevista. Se o domicílio foi classificado como inseguro, todos os membros foram pesados e medidos. A prevalência de insegurança alimentar para todas as famílias foi de 11\% (IC95\%: 9-13). Nas famílias que vivem em insegurança coexistem por um lado, o excesso de peso e obesidade entre as crianças e os adultos $e$, por outro, uma prevalência substancial de déficit de crescimento entre as crianças (21\%). Conclui-se que esse estado de insegurança alimentar pode estar relacionado não somente à diminuição da quantidade de alimentos como à perda da qualidade nutritiva.

Hábitos Alimentares; Estado Nutricional; Segurança Alimentar e Nutricional

\section{Colaboradores}

J. V. Santos participou do projeto, revisão bibliográfica, elaboração do trabalho de campo, análise dos dados, discussão dos resultados e redação do manuscrito. D. P. Gigante orientou a realização de todas as etapas da pesquisa e revisou o artigo. M. R. Domingues trabalhou na revisão da análise de dados e redação final.

\section{Agradecimentos}

A Coordenação de Aperfeiçoamento de Pessoal de Nível Superior (CAPES) pelo apoio financeiro. 


\section{Referências}

1. Ministério da Saúde. Estudo de caso Brasil: a in tegração das ações de alimentação e nutrição nos planos de desenvolvimento nacional para o alcance das metas do milênio no contexto do direito humano a alimentação adequada. Brasília: Ministério da Saúde; 2005.

2. Bickel GW, Nord M, Price C, Hamilton W, Cook J. Measuring food security in the United States. Guide to measuring household food security. Revised 2000. Alexandria: United States Department of Agriculture; 2000.

3. Campbell CC. Food insecurity: a nutritional outcome or a predictor variable? J Nutr 1991; 121: 408-15.

4. Conselho Nacional de Segurança Alimentar e Nutricional. Documento base - III Conferência Nacional de Segurança Alimentar e Nutricional. Brasília: Conselho Nacional de Segurança Alimentar e Nutricional; 2007.

5. Panigassi G, Segall-Corrêa AM, Marin-León L, Pérez-Escamilla R, Sampaio MFA, Maranha LK. Insegurança alimentar como indicador de iniqüidade: análise de inquérito populacional. Cad Saúde Pública 2008; 24:2376-84.

6. Kendall A, Olson CM, Frongillo Jr. EA. Validation of the Radimer/Cornell measures of hunger and food insecurity. J Nutr 1995; 125:2793-801.

7. Harrison GG, Stormer A, Herman DR, Winham DM. Development of a Spanish-language version of the U.S. household food security survey module. J Nutr 2003; 133:1192-7.

8. Melgar-Quinonez H, Zubieta AC, Valdez E, Whitelaw B, Kaiser L. Validation of an instrument to monitor food insecurity in Sierra de Manantlan, Jalisco. Salud Pública Méx 2005; 47:413-22.

9. Albert PL, Sanjur D. The adaptation and validation of the food security scale in a community of Caracas, Venezuela. Arch Latinoam Nutr 2000; 50: 334-40.

10. Alvarez MC, Estrada A, Montoya EC, Melgar-Quinonez $\mathrm{H}$. Validation of a household food security scale in Antioquia, Colombia. Salud Pública Méx 2006; 48:474-81.

11. Frongillo Jr. EA. Validation of measures of food insecurity and hunger. J Nutr 1999; 129(2S Suppl):506S-9S

12. Perez-Escamilla R, Segall-Correa AM, Kurdian-Maranha L, Sampaio MMF, Marin-Leon L, Panigassi G. An adapted version of the U.S. Department of Agriculture Food Insecurity module is a valid tool for assessing household food insecurity in Campinas, Brazil. J Nutr 2004; 134:1923-8.

13. Instituto Brasileiro de Geografia e Estatística. Pesquisa Nacional por Amostra de Domicílios. Segurança alimentar 2004. Rio de Janeiro: Instituto Brasileiro de Geografia e Estatística; 2006.

14. Ministério da Saúde. Pesquisa Nacional de Demografia e Saúde da Criança e da Mulher - PNDS 2006. Brasília: Ministério da Saúde; 2008.

15. Barria RM, Amigo H. Nutrition transition: a review of Latin American profile. Arch Latinoam Nutr 2006; 56:3-11.
16. Instituto Brasileiro de Geografia e Estatística. Pes quisa Nacional de Orçamentos Familiares 20022003 Análise da disponibilidade domiciliar de alimentos e do estado nutricional do Brasil. Rio de Janeiro: Instituto Brasileiro de Geografia e Estatística; 2004

17. Instituto Brasileiro de Geografia e Estatística. Estado nutricional de crianças e adolescentes. Pesquisa de Orçamentos Familiares 2002-2003. Rio de Janeiro: Instituto Brasileiro de Geografia e Estatística; 2004

18. Lohman TG, Roche AF, Martorell R. Anthropometric standardization reference manual. Champaign: Human Kinetics Books; 1986.

19. Blumberg SJ, Bialostosky K, Hamilton WL, Briefel $\mathrm{RR}$. The effectiveness of a short form of the Household Food Security Scale. Am J Public Health 1999; 89:1231-4.

20. World Health Organization. WHO Multicentre Growth Reference Study Group. WHO child growth standards: length/height-for-age, weight-for-age, weight-for-length, weight-for-height and body mass index-for-age: methods and development. Geneva: World Health Organization; 2006.

21. World Health Organization. Global database on body mass index. http://www.who.int/bmi/index. jsp (acessado em 15/Nov/2008).

22. Onis M, Onyango AW, Borghi E, Siyam A, Nishida C, Siekmann J. Development of a WHO growth reference for school-aged children and adolescents. Bull World Health Organ 2007; 85:660-7.

23. Dinour LM, Bergen D, Yeh MC. The food insecurity-obesity paradox: a review of the literature and the role food stamps may play. J Am Diet Assoc 2007; 107:1952-61.

24. Power E. Individual and household food insecurity in Canada: position of Dietitians of Canada. Can J Diet Pract Res 2005; 66:43-6.

25. Flegal KM, Carroll MD, Ogden CL, Johnson CL. Prevalence and trends in obesity among US adults, 1999-2000. JAMA 2002; 288:1723-7.

26. Ogden CL, Flegal KM, Carroll MD, Johnson CL. Prevalence and trends in overweight among US children and adolescents, 1999-2000. JAMA 2002; 288:1728-32.

27. Laraia BA, Siega-Riz AM, Evenson KR. Self-reported overweight and obesity are not associated with concern about enough food among adults in New York and Louisiana. Prev Med 2004; 38:175-81.

28. Barroso GS, Sichieri R, Salles-Costa R. Factors associated with malnutrition in children living in food insecurity households. Rev Bras Epidemiol 2008; 11:484-94.

29. Gigante DP, Dias-da-Costa JS, Olinto MTA, Menezes AMB, Macedo S. Obesidade da população adulta de Pelotas, Rio Grande do Sul, Brasil e associação com nível sócio-econômico. Cad Saúde Pública 2006; 22:1873-9.

Recebido em 14/Abr/2009

Versão final reapresentada em 07/Ago/2009

Aprovado em 21/Out/2009 